\title{
Utilização do aplicativo "Waze" no cálculo de distância através da integral definida
}

"Waze" app utilization in distance calculation through definite integral

$$
\text { Leila Thomazelli Thieghi*1] }
$$

${ }^{1}$ Universidade Federal de São Paulo, Instituto de Ciências Ambientais, Químicas e Farmacêuticas, Departamento de Física, Diadema, SP, Brasil.

Recebido em 19 de janeiro de 2021. Revisado em 23 de julho de 2021. Aceito em 16 de agosto de 2021.

\begin{abstract}
Este trabalho apresenta os resultados de uma atividade prática em grupo de Cálculo Integral, centrada nos conceitos intuitivos de velocidade escalar e integral definida, a fim de encontrar uma distância total percorrida. O processo de construção foi realizado com uma vivência de coleta de dados de velocidade em função do tempo durante um percurso pré-definido com a utilização de um aplicativo para smartphones, "Waze". Os dados foram analisados pelos estudantes visando aproximar o valor da integral definida pela soma de áreas em um gráfico de velocidade escalar em função do tempo para obter a distância total percorrida. O resultado da atividade foi de grande utilidade para a compreensão da estimativa do valor de uma integral definida por parte dos estudantes. Palavras-chave: Cálculo. Integral Definida. Aplicativo para smartphone. Distância.
\end{abstract}

This paper presents results of a practical and group activity of Integral Calculus, centered on intuitive concepts of speed and definite integral. The construction process was carried out with an experience of collecting speed data as a function of time during a pre-defined route using a smartphone application, "Waze". The data were analyzed by students to approximate the value of the definite integral by adding areas in a graph of speed as a function of time to obtain the total distance covered. The result of the activity was very useful for the estimation of the value of a definite integral by the students.

Keywords: Calculus. Definite Integral. Smartphone applets. Distance.

\section{Introdução}

O Cálculo Diferencial e Integral constitui-se em uma área de conhecimento relevante para a cultura científica moderna, principalmente, pela potencialidade de sua utilização na resolução de problemas de distintas áreas de formação de estudantes de ensino superior, sendo por essa razão muito importante o estudante dominar os conceitos envolvidos e saber empregá-los corretamente. No ensino de Física, principalmente, as ferramentas matemáticas de Cálculo Diferencial e Integral se fazem indispensáveis, e muitas vezes o insucesso do estudante nas matérias de Física é devido à dificuldade de compreensão dos conceitos de Cálculo, o que indica que devemos nos preocupar em transmitir com clareza esses conceitos. Nos últimos anos, o processo de ensino e aprendizagem do Cálculo Diferencial e Integral passou a ser objeto de várias pesquisas na educação superior devido às dificuldades de compreensão de seus conceitos, ao elevado índice de evasão e reprovação dos discentes, elementos presentes desde o início do ensino de Cálculo Integral e Diferencial no Brasil, como Lima [1] apresenta, reportando inclusive como se deu a transição da disciplina de Análise Matemática para Cálculo na Universidade

\footnotetext{
*Endereço de correspondência: leila.thieghi@unifesp.br
}

de São Paulo. Naquele tempo [2], na universidade, era ignorada toda e qualquer necessidade de adaptação de materiais ou conteúdo, sendo as dificuldades atribuídas às falhas no trabalho do aluno.

Ultimamente esse pensamento foi abandonado pela maioria dos docentes, e dia após dia, mais e mais docentes concordam [1] que a aprendizagem seria mais exitosa se houvesse uma relação de parceria entre professores e estudantes universitários na construção do conhecimento que está sendo trabalhado, com reflexões conjuntas a respeito do processo de ensino e de aprendizagem na universidade. Junto a esse pensamento, veio a inclusão digital e uso de ferramentas tecnológicas no ensino, já que os jovens são extremamente atraídos por tablets e smartphones. No entanto, usar ferramentas que vão além de um vídeo na internet, ou visitas a sites para pesquisa, por exemplo, ainda constitui um desafio para a grande maioria dos docentes em atuação por falta de tempo e/ou de recursos [3]. Muitos trabalhos na área de Física com a utilização de smartphones, tablets, aplicativos e realidade virtual já podem ser encontrados, tanto no Ensino Fundamental e Médio [4 7], quanto no ensino superior [8 11], e esse número tende a aumentar no período pós-pandemia [12. Alguns trabalhos têm sido realizados também unindo matemática e situações de Cinemática com bastante êxito no processo 
ensino-aprendizagem [13 16, uma vez que a Cinemática é parte do dia a dia das pessoas. Tendo como objetivo unir o ensino de Cálculo com um problema simples de Física, criamos uma atividade prática extraclasse com estudantes ingressantes do Curso de Química da Universidade Federal de São Paulo (UNIFESP) - Campus Diadema, com a utilização do aplicativo "Waze" para smartphones. A escolha do aplicativo se deu por vários fatores, entre eles ser um método seguro, padronizado e sem qualquer custo aos estudantes, já que qualquer pessoa pode baixar gratuitamente em um smartphone que tenha sistema GPS. O aplicativo "Waze" é utilizado por motoristas que desejam encontrar a rota mais rápida entre dois pontos, e disponibiliza várias funcionalidades, entre elas a de medir em tempo real a velocidade escalar instantânea do veículo (que carregue o smartphone dentro dele).

A atividade foi desenvolvida na disciplina de Cálculo I, que aborda tanto as derivadas quanto as integrais de funções de uma variável. O conceito da integral definida, o primeiro tópico estudado em integrais, é dado como o limite da soma de áreas retangulares sob o gráfico de uma função positiva no intervalo de integração, através da famosa soma de Riemann. O docente pode perceber durante a aula expositiva que os estudantes entendem a ideia intuitiva desta soma, mas se confundem muito quando precisam estimar o valor de uma integral definida usando um número finito de subdivisões do intervalo de integração. Outra confusão muito frequente é acreditarem que uma integral definida sempre calcula uma área, não fazendo associação com o significado físico daquela área.

O objetivo da atividade proposta foi mostrar ao aluno uma aplicação simples e direta do cálculo de integral definida, em um contexto de cálculo de distância total percorrida. Essa distância foi calculada pelo valor aproximado de uma integral definida através da soma de áreas, fazendo a conexão direta entre a soma de Riemann e a integral definida.

Sabemos que a área $A$ de uma região sob o gráfico de uma função, $f(t)$ contínua em $[a, b]$ e delimitada pelas retas $t=a$ e $t=b$, é igual ao limite da soma das áreas dos retângulos aproximadores, quando o número $n$ de retângulos tende a infinito:

$$
\begin{aligned}
A & =\lim _{n \rightarrow \infty}\left[f\left(t_{1}^{*}\right) \triangle t_{1}+f\left(t_{2}^{*}\right) \triangle t_{2}+\cdots+f\left(t_{n}^{*}\right) \triangle t_{n}\right] \\
& =\lim _{n \rightarrow \infty} \sum_{i=1}^{n} f\left(t_{1}^{*}\right) \triangle t_{i}
\end{aligned}
$$

onde $t_{i}^{*}$ é o ponto amostral, $\triangle t_{i}$ é a largura de cada retângulo aproximador, e $f\left(t_{i}^{*}\right)$ é a respectiva altura. Os pontos amostrais $t_{i}^{*}$ podem ser quaisquer pontos dentro dos subintervalos $\triangle t_{i}$, como mostra a Figura 1 usualmente, usamos as extremidades direita ou esquerda dos subintervalos, porém pode-se utilizar também o ponto médio de cada subintervalo, obtendo com essa última escolha a melhor aproximação para a integral.

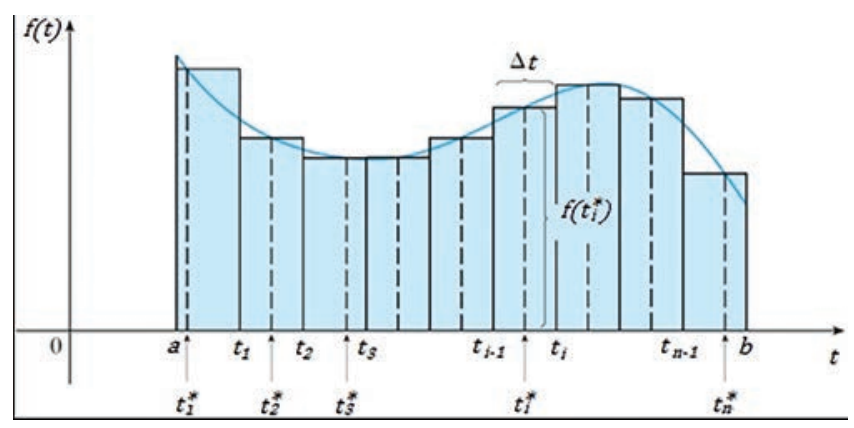

Figura 1: A figura mostra como aproximar a área sob a curva entre os pontos a e $b$ pela soma dos retângulos aproximadores de base $\triangle \boldsymbol{t}_{\boldsymbol{i}}$ e altura $\boldsymbol{f}\left(\boldsymbol{t}_{\boldsymbol{i}}^{*}\right)$, valor de $f$ em um número qualquer $\boldsymbol{t}_{\boldsymbol{i}}^{*}$ no $i$-ésimo subintervalo. (figura adaptada de STEWART, J. [17])

Costumeiramente consideramos que todos os retângulos tenham a mesma largura, ou seja, que $\triangle t_{i}=\triangle t=\frac{b-a}{n}$, sendo então $t_{i}=a+i \triangle t$, porém os retângulos podem ter larguras distintas entre si, já que todas essas larguras tenderão a zero no limite quando $n$ tender a infinito.

$\mathrm{O}$ último termo que aparece à direita na Equação (1) calcula o limite da soma de Riemann quando $n$ tende a infinito, e é a própria definição da integral definida da função $f(t)$ no intervalo $[a, b]$, ou seja

$$
\int_{a}^{b} f(t) d t=\lim _{n \rightarrow \infty} \sum_{i=1}^{n} f\left(t_{i}^{*}\right) \triangle t
$$

Sabemos que velocidade é uma grandeza vetorial, com módulo, direção e sentido, e que seu módulo representa a velocidade escalar. Sabemos também que velocidade escalar indica a variação do espaço, $S$, em função do tempo, $t$, e assim podemos escrever a velocidade escalar média como

$$
v_{m}=\frac{\triangle S}{\triangle t}
$$

onde $v_{m}$ é velocidade escalar média, $\triangle S$ é a variação do espaço e $\triangle t$ é a variação do tempo. A velocidade escalar instantânea, $v$, pode ser obtida pelo limite da velocidade média quando a variação do tempo tende a zero, isto é

$$
v=\lim _{\triangle t \rightarrow 0} v_{m}=\lim _{\triangle t \rightarrow 0} \frac{\triangle S}{\triangle t}=\frac{d S}{d t}
$$

ou seja, a velocidade escalar instantânea pode ser calculada através da derivada do espaço em relação ao tempo.

Então, se tivermos um gráfico da função velocidade escalar instantânea em função do tempo, $v(t)$, a área, $A$, sob a curva entre $a$ e $b$ será numericamente igual à integral

$$
A=\int_{a}^{b} v(t) d t
$$


Utilizando o Teorema Fundamental do Cálculo, sabemos que

$$
\int_{a}^{b} v(t) d t=\int_{a}^{b}\left(\frac{d S}{d t}\right) d t=S(b)-S(a)
$$

A Equação (6) diz que a integral da velocidade escalar instantânea entre os instantes $a$ e $b$ é igual à variação do espaço entre os instantes $a$ e $b$, que também é igual à distância total percorrida no intervalo temporal de $a$ até $b$, desde que tenhamos $v(t) \geq 0$ em $[a, b]$, e então podemos escrever que

$$
\text { distância entre a e } b=\int_{a}^{b} v(t) d t
$$

Na prática não é possível fazermos $n$ tender a infinito, porém podemos aproximar o valor da integral pela área sob a curva se utilizarmos um número grande de subintervalos. A proposta da atividade foi utilizar diferentes números de subintervalos para calcular uma mesma distância.

Daqui em diante, para simplificar a leitura, utilizaremos o termo velocidade para nos referirmos à velocidade escalar instantânea.

\section{Atividade Proposta e Coleta de Dados}

A atividade extraclasse consistiu em solicitar que os estudantes calculassem a distância percorrida entre duas unidades do Campus Diadema da UNIFESP, no percurso do ônibus (gratuito) do Campus, através de coleta de dados de velocidade em função do tempo. Como o percurso dos ônibus é fixo, independentemente de dia ou horário, os estudantes poderiam realizar a atividade em quaisquer dias e horários livres, e todos os estudantes coletariam dados de um único percurso.

Para as medidas de velocidades os estudantes deveriam utilizar seus próprios smartphones, com o aplicativo "Waze", na opção "mostrar velocímetro" (Figura 2). Os estudantes não necessitavam pedir para o "Waze" traçar o trajeto, apenas usar o GPS para as medidas de velocidade do ônibus. Já para as medidas de tempo, deveriam utilizar um cronômetro qualquer (de um relógio digital ou um cronômetro de smartphone), iniciado no momento de partida do ônibus, e parado apenas ao término da rota. Ênfase foi dada no fato de que não havia necessidade de parar o cronômetro nas paradas intermediárias do ônibus! Se fosse momento de coleta de dados, deveriam anotar velocidade igual a zero quando o ônibus estivesse parado.

Os estudantes deveriam se dividir em grupos de acordo com suas afinidades, de modo que o número de integrantes fosse igual ao número de intervalos de tempo utilizados. Os métodos de medidas de velocidade poderiam ser diversos, por exemplo, a cada 2 minutos, a cada 30 segundos, a cada veículo de cor vermelha que passasse pela janela do ônibus, a cada buzina que fosse ouvida, etc. Os diferentes métodos de medidas de velocidades iriam impactar diretamente nos intervalos de tempo, de maneira a trabalharem tanto intervalos de tempo idênticos como também diferentes. Cada membro, com seu intervalo de tempo escolhido, deveria gerar uma tabela de dados de velocidade em função do tempo, para diferentes instantes durante o trajeto.

A distância real entre as duas unidades no percurso do ônibus deveria ser anotada pelas leituras no odômetro do ônibus ao início e ao final da viagem, para comparação final com os resultados calculados pelos estudantes.

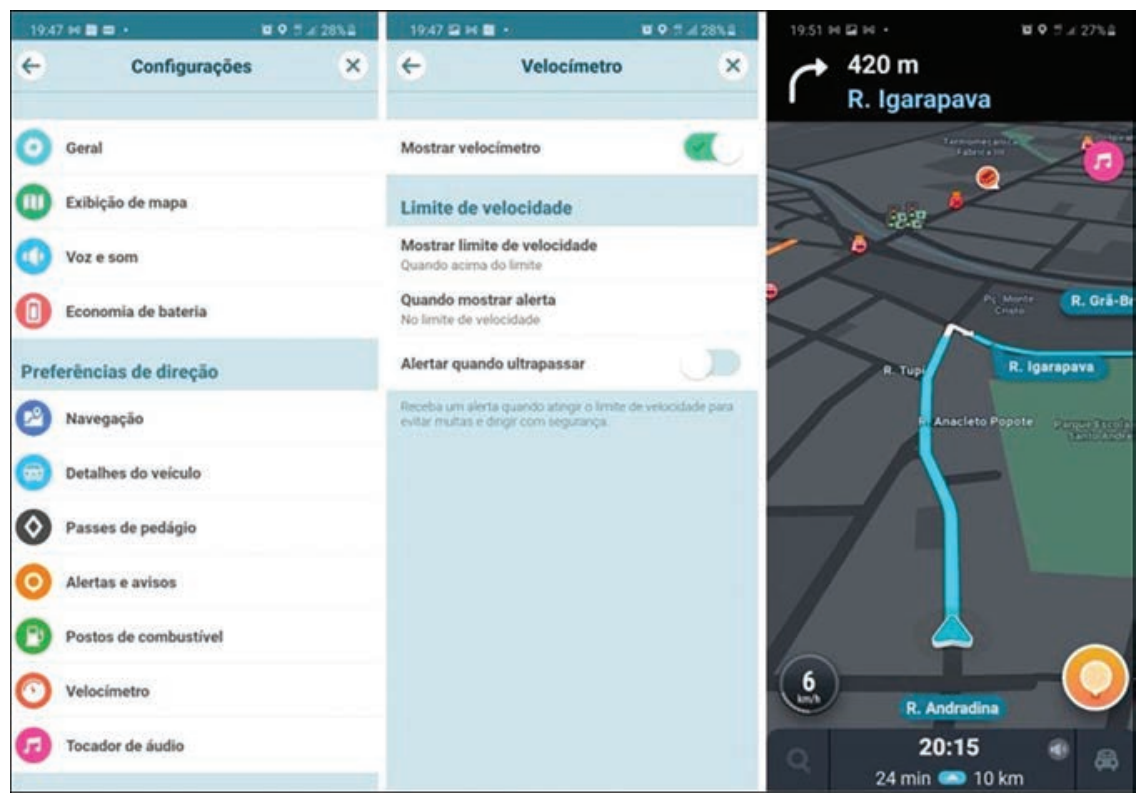

Figura 2: Captura de tela do "Waze", mostrando na primeira tela as opções de configurações, na segunda tela a opção de mostrar velocímetro, e na terceira tela o velocímetro abaixo à esquerda da tela. 
Os dados foram coletados durante trajeto do ônibus circular da UNIFESP DIADEMA, no trajeto Conforja Florestan, cuja distância total percorrida é de $2,5 \mathrm{~km}$ e costumeiramente dura cerca de 10 minutos, sem paradas intermediárias para subida ou descida de passageiros.

As seguintes instruções foram passadas aos estudantes:

Cada membro deve tratar seus dados individualmente e construir um gráfico de dispersão de velocidade em função do tempo.

Decidir qual será o ponto amostral em cada subintervalo: extremo esquerdo, extremo direito ou ainda o ponto médio de cada subintervalo.

Desenhar os retângulos aproximadores, deixando claro qual o ponto amostral utilizado.

Calcular a área de cada retângulo aproximador pela multiplicação do intervalo de tempo pela velocidade no ponto amostral escolhido, certificando-se que seus dados de velocidade e tempo estejam em unidades compativeis (por exemplo $\mathrm{m} / \mathrm{s}$ e $\mathrm{s})$; caso contrário, fazer as conversões necessárias.

Realizar a soma das áreas de todos os retângulos aproximadores, obtendo assim uma aproximação para a distância total percorrida pelo ônibus.

Comparar o resultado obtido com o valor real anotado do odômetro.

Discutir os resultados obtidos pelo grupo em função dos intervalos de tempos entre medições.

\section{Tratamento Inicial dos Dados pelos Discentes}

Cada integrante do grupo foi responsável pelo tratamento de seus dados, usando a ferramenta Excel, disponível aos estudantes nos computadores das salas de informática da UNIFESP - Campus Diadema. No Excel, deveriam criar duas colunas de dados, tempo no eixo das abcissas (eixo horizontal) e velocidade no eixo das ordenadas (eixo vertical). Os dados deveriam ser plotados em um gráfico de dispersão; isso foi tudo o que deveriam realizaram com o Excel, exigindo quase nenhum conhecimento do programa. Se o aluno tivesse alguma dificuldade nesse processo, poderia ser feito manualmente, desde que as escalas fossem colocadas corretamente. A partir desse ponto, o trabalho de calcular as áreas deveria ser manual. Cuidado deveria ser tomado com as unidades de medida na hora dos cálculos, para que estivessem em sistemas compatíveis (velocidade em $\mathrm{m} / \mathrm{s}$ e tempo em $\mathrm{s}$, ou velocidade $\mathrm{em} \mathrm{km} / \mathrm{h}$ e tempo em h, por exemplo).

\section{Resultados Parciais Obtidos pelos Estudantes}

Os diferentes grupos coletaram seus dados em diferentes dias e horários, e gráficos de velocidade em função do tempo são mostrados para os cinco grupos, com o menor passo de tempo utilizado por cada grupo. Pode-se ver facilmente na Figura 3 que os grupos de 1 a 4 seguem um padrão parecido, com velocidades quase sempre abaixo de $40 \mathrm{~km} / \mathrm{h}$ e vários pontos de parada, possivelmente correspondentes a semáforos vermelhos. O grupo 5 apresentou um padrão diferente, com velocidades várias
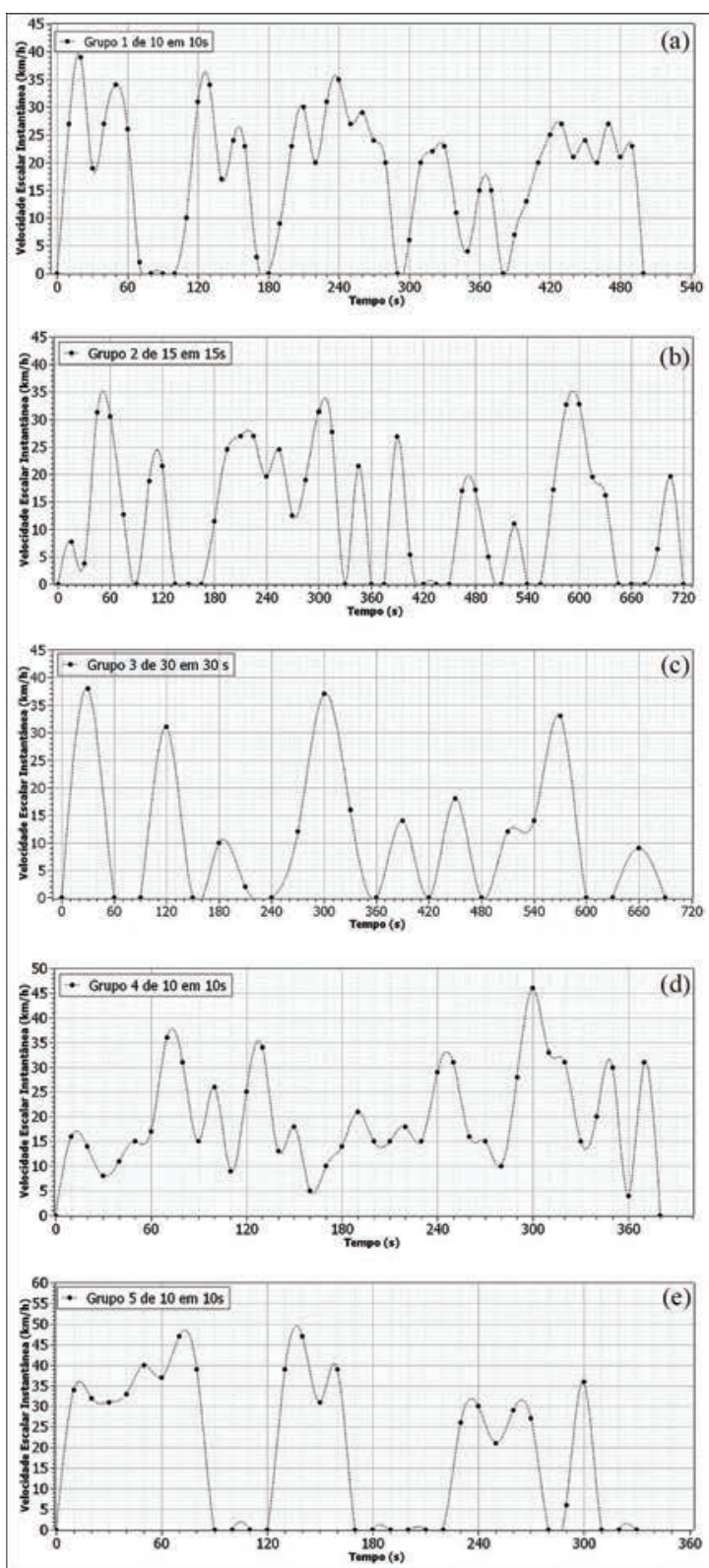

Figura 3: Gráficos de velocidade em função do tempo para os grupos 1(a), 2(b), 3(c), 4(d) e 5(e), para os menores passos de tempo utilizados. As curvas tracejadas servem apenas de guia para os olhos!. 
Tabela 1: Resultados das distâncias percorridas calculadas pelos grupos, em diferentes intervalos de tempo.

\begin{tabular}{|c|c|c|c|c|}
\hline Grupo & $\begin{array}{l}\text { Intervalo de tempo } \\
\text { entre cada medida }\end{array}$ & $\begin{array}{c}\text { Número de } \\
\text { pontos coletados }\end{array}$ & $\begin{array}{l}\text { Distância total } \\
\text { calculada }(\mathrm{km})\end{array}$ & Observações \\
\hline \multirow[t]{4}{*}{1} & $10 \mathrm{~s}$ & 49 & 2,52 & \multirow{4}{*}{$\begin{array}{l}\text { tomaram dados em } \mathrm{t}=0 \text { e usaram as } \\
\text { extremidades esquerdas dos intervalos }\end{array}$} \\
\hline & $30 \mathrm{~s}$ & 17 & 2,58 & \\
\hline & $40 \mathrm{~s}$ & 14 & 2,79 & \\
\hline & $1 \mathrm{~min}$ & 8 & 2,75 & \\
\hline \multirow[t]{4}{*}{2} & $15 \mathrm{~s}$ & 47 & 2,50 & \multirow{4}{*}{$\begin{array}{l}\text { não tomaram dados em } \mathrm{t}=0 \text { e usaram as } \\
\text { extremidades direitas dos intervalos }\end{array}$} \\
\hline & $30 \mathrm{~s}$ & 23 & 2,29 & \\
\hline & $45 \mathrm{~s}$ & 15 & 2,12 & \\
\hline & $1 \mathrm{~min}$ & 11 & 2,74 & \\
\hline \multirow[t]{4}{*}{3} & $30 \mathrm{~s}$ & 24 & 2,05 & \multirow{4}{*}{$\begin{array}{l}\text { tomaram dados em } \mathrm{t}=0 \text { e usaram as } \\
\text { extremidades esquerdas dos intervalos }\end{array}$} \\
\hline & $1 \mathrm{~min}$ & 13 & 2,40 & \\
\hline & $1,5 \mathrm{~min}$ & 8 & 2,68 & \\
\hline & $2 \min$ & 6 & 2,86 & \\
\hline \multirow[t]{4}{*}{4} & $10 \mathrm{~s}$ & 37 & 2,06 & \multirow{4}{*}{$\begin{array}{l}\text { usaram as extremidades esquerdas dos } \\
\text { intervalos }\end{array}$} \\
\hline & $30 \mathrm{~s}$ & 25 & 2,77 & \\
\hline & $1 \mathrm{~min}$ & 13 & 3,48 & \\
\hline & $1,5 \min$ & 8 & 1,52 & \\
\hline \multirow[t]{3}{*}{5} & $10 \mathrm{~s}$ & 34 & 1,73 & \multirow{3}{*}{$\begin{array}{l}\text { usaram as extremidades esquerdas dos } \\
\text { intervalos }\end{array}$} \\
\hline & $30 \mathrm{~s}$ & 12 & 1,60 & \\
\hline & $1 \mathrm{~min}$ & 6 & 1,72 & \\
\hline
\end{tabular}

vezes acima de $40 \mathrm{~km} / \mathrm{h}$, menos paradas, porém mais demoradas que as paradas dos demais grupos. Também pode ser visto que os trajetos duraram por volta de 12 minutos, com exceção dos grupos 1 (aproximadamente $8,5 \mathrm{~min}$ ) e 5 (aproximadamente $5,5 \mathrm{~min}$ ).

A Tabela 1 apresenta os resultados dos cinco grupos que concluíram a atividade.

Cada grupo apresentou seus resultados de distância calculada em função do intervalo de tempo entre medições e com eles as conclusões parciais, antes da discussão de todos os resultados em sala de aula:

GRUPO 1: "Sabendo que a integral é definida pela somatória das áreas dos retângulos formados, percebemos que a medida que o número de retângulos no gráfico aumenta consequentemente o valor da largura de cada retângulo diminui, o que implica em uma estimativa mais precisa da real distância do trajeto. Quando utilizamos intervalos de tempo de 60 em 60 segundos, vimos que a distância percorrida foi de aproximadamente 2,7 quilômetros, e já quando escolhemos intervalos menores, $10 \mathrm{em} 10$ segundos, foi de aproximadamente 2,5 quilômetros, representando um valor mais próximo do real."

GRUPO 2: "Nota-se que, quanto mais marcações forem feitas, mais próximo do valor real é o resultado. Provando que, o Limite da somatória das marcações com " $n$ " (número de marcações) tendendo a infinito, é o valor mais próximo possível do resultado real.
Obs: A distância encontrada pelo grupo foi de 2,5 Km."

GRUPO 3: "Quanto menor o valor de medição (os minutos), mais próximo foi o resultado da distância percorrida em relação ao seu valor real. Isso ocorre pois à medida em que diminuímos os intervalos de tempo, aumentamos a quantidade de retângulos que representariam um gráfico de uma função qualquer, e dessa maneira, a soma das áreas das barras fica mais próxima da área real de uma curva."

Os grupos 4 e 5 não apresentaram nenhuma conclusão.

\section{Análise dos Resultados Parciais}

Analisando os resultados obtidos pelos grupos a partir da Tabela 1 nota-se que nem sempre os menores intervalos de tempo entre medições (que correspondem a um maior número de retângulos aproximadores) forneceram a melhor aproximação da distância percorrida pelo ônibus; na verdade isso ocorreu apenas para os grupos 1 (10 s) e 2 (15 s), enquanto que para os grupos 3 (1 min) e 4 (30 s) a melhor aproximação ocorreu com o segundo menor intervalo de tempo entre medições, ao passo que o grupo 5 obteve a melhor aproximação para o menor intervalo (10 s), porém com resultados muito discrepantes do esperado. Na Figura 3 é possível notar que o grupo 5 apresentou um padrão de velocidades muito distinto dos demais grupos: o percurso durou menos tempo em comparação aos demais grupos, as velocidades ultrapassaram os $40 \mathrm{~km} / \mathrm{h}$ várias vezes, e 
as paradas foram menos frequentes e mais longas. Dessa forma, acredita-se que o grupo 5 não coletou os dados nas mesmas condições que os demais, talvez fazendo um percurso diferente, e mais curto, em automóvel.

Matematicamente, não há dúvida alguma quanto à melhora da aproximação do valor da integral definida quanto maior o número de retângulos aproximadores, e pode-se constatar isso facilmente em um exercício simples, por exemplo, aproximando o valor da integral de $f(x)=x^{2}$ entre 0 e 1 , usando 2 retângulos aproximadores, depois 4, 8 e 16. Analogamente, no caso de estimar a distância percorrida pela aproximação da integral de uma função velocidade em função do tempo, aumentar o número de retângulos aproximadores irá proporcionar uma melhor aproximação da distância, o que equivale a diminuir o intervalo de tempo entre os retângulos (diminuir a largura dos retângulos).

Quando parte-se para uma abordagem experimental, é preciso levar em conta todo o processo de planejamento do experimento e obtenção dos dados experimentais. A atividade como foi realizada por esses alunos pode ser considerada quase-experimental [16] e por essa razão não envolveu análise estatística dos dados, porém se a atividade tiver a proposta de ser um experimento em Física, é preciso uma análise adicional. A maneira adequada de mostrar que a qualidade da estimativa da integral pela somatória melhora quando se usa passos menores de tempo seria comparar as dispersões de valores obtidos nessas estimativas de acordo com o passo utilizado. Assim, sugere-se uma readequação da proposta, permitindo comparar as dispersões dos valores de distância percorrida, o que implica em usar valores de passo de tempo pré-estabelecidos e comuns a todos os grupos, conforme exposto a seguir.

\section{Aperfeiçoamento da Atividade Proposta}

Para que seja possível comparar as dispersões de valores obtidos por todos os grupos nas estimativas de distância percorrida de acordo com o passo utilizado, é sugerido padronizar os intervalos de tempo entre medidas para todos os grupos, adicionando a instrução "Todos os grupos deverão trabalhar com intervalos iguais de tempo de coleta de dados, sendo os passos sempre múltiplos do menor passo adotado". Com essa modificação torna-se possível fazer uma análise com os dados obtidos pelos distintos grupos com o mesmo intervalo de tempo entre medidas.

A seguir é realizada uma análise comparativa para fins de ilustração, apresentando os resultados que seriam obtidos se uma análise desse tipo fosse feita com os dados brutos que foram obtidos pelos estudantes e entregues ao docente.

O intervalo de $30 \mathrm{~s}$ foi o menor intervalo comum a todos os grupos, então foi adotado como o menor passo a ser utilizado. Todos os grupos realizaram medidas a cada minuto, então o segundo passo da nova análise foi de $60 \mathrm{~s}$. Apenas dois grupos realizaram medidas a cada minuto e meio, porém como os estudantes entregaram suas planilhas de dados, foi possível utilizar as medidas a cada $30 \mathrm{~s}$, selecionando os dados de $3 \mathrm{em} 3$, ou usar as medidas a cada $45 \mathrm{~s}$ selecionando os dados de dois em dois, de modo que o terceiro passo de tempo utilizado foi de 90 s. Esse procedimento foi possível porque ao verificar os dados de cada grupo, era perceptível que todos os integrantes tomaram os dados nas mesmas condições, ou seja, estavam todos dentro do mesmo ônibus ao mesmo tempo, já que os valores de velocidade em tempos iguais coincidiam. Analogamente, o quarto intervalo de tempo na nova análise passou a ser de $120 \mathrm{~s}$.

De posse dos dados brutos de velocidade em função do tempo foi possível a construção de histogramas de velocidades para os cinco grupos. Optou-se pela utilização dos dados de velocidade na menor escala em que foram efetivamente medidos, uma vez que o uso dos tempos 30,60 , 90 e 120 s foi apenas para possibilitar avaliar a dispersão entre os diversos grupos, sendo que a maior informação possível sobre a distribuição de velocidades do trajeto é a obtida no menor passo de tempo. Esses histogramas estão na Figura 4 cada um com seu desvio padrão, $\sigma$. Com exceção do grupo 4 , vê-se claramente picos de velocidades baixas (entre $0 \mathrm{e} 4 \mathrm{~km} / \mathrm{h}$ ), devido às paradas no trecho urbano em que a atividade ocorreu. Também é interessante notar que uma vez mais o grupo 5 se destaca entre os demais, com um valor mais elevado de desvio padrão, evidenciando a diferença do comportamento da velocidade em função do tempo desse grupo em comparação aos demais. Os histogramas para os intervalos de tempo entre medições utilizados no aperfeiçoamento da proposta (30, 60, 90 e 120 s) encontram-se no Material Suplementar 1.

A Figura 5 mostra, para o grupo 1, os retângulos aproximadores desenhados para todos os intervalos de tempo (30, 60, 90 e 120 s). As larguras dos retângulos correspondem ao intervalo de tempo entre medições consecutivas de velocidades, enquanto que as alturas correspondem aos valores de velocidades nos pontos amostrais selecionados. Os resultados para os demais grupos encontram-se no Material Suplementar 1. O grupo 2 foi o único a utilizar as extremidades direitas dos subintervalos; os demais grupos utilizaram as extremidades esquerdas.

Na Tabela 2, apresentamos os resultados calculados de distâncias percorridas, em quilômetros, dos cinco grupos, com intervalos de tempo padronizados em 30, 60, 90 e 120 s. A diferença entre a Tabela 2 e a Tabela 1 é apenas que na Tabela 2 os intervalos de tempo estão padronizados para todos os grupos, porém ainda são resultados obtidos grupo a grupo.

Observando os resultados da Tabela 2 ainda pode ser visto que o resultado esperado pela Equação (2) se cumpriu apenas para os grupos 1,2 e 4, ou seja, apenas esses três grupos tiveram o valor calculado de distância 

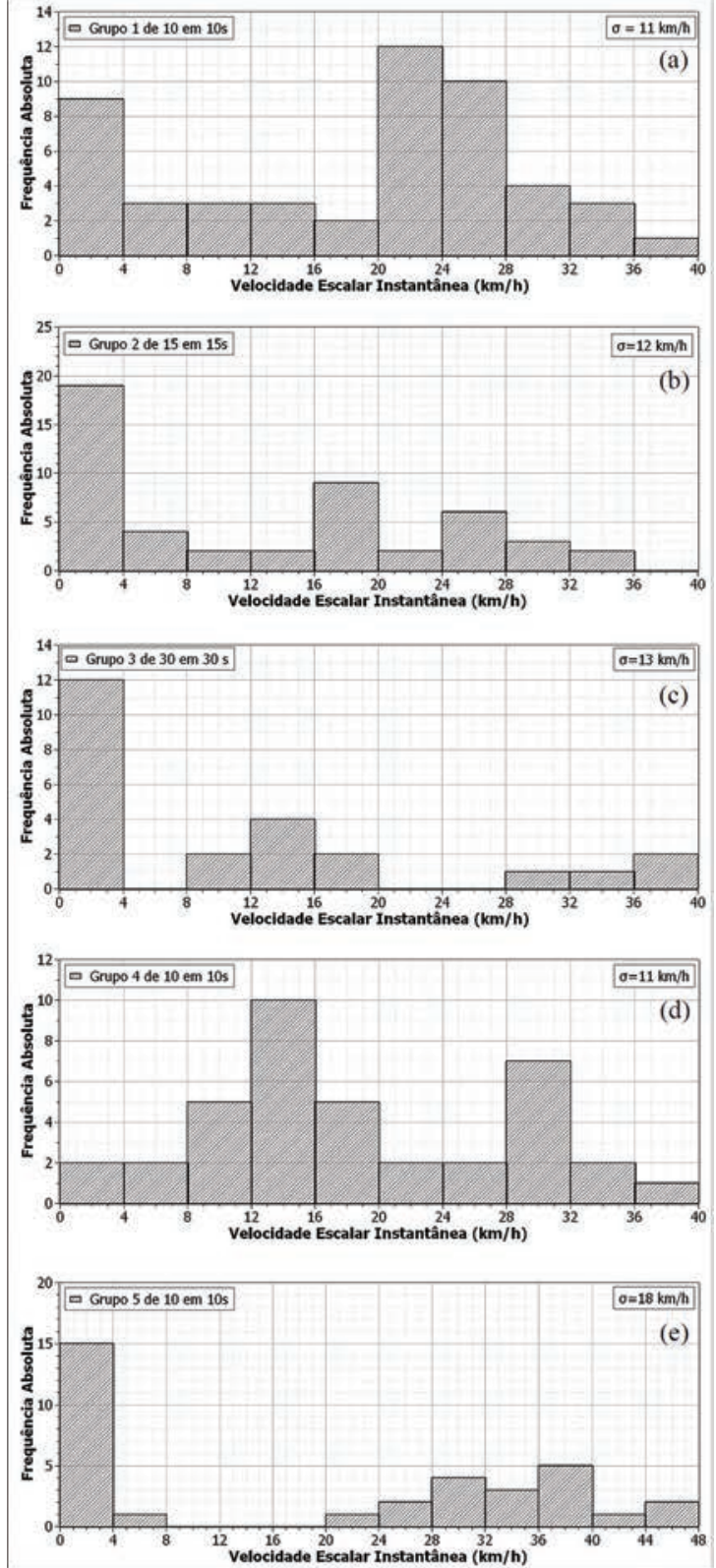

Figura 4: Histogramas de velocidades para os menores passos de tempo utilizados para os grupos 1(a), 2(b), 3(c), 4(d) e 5(e).

percorrida mais próximo do valor real quando utilizaram um número maior de retângulos aproximadores (ou menor intervalo de tempo (30 s) entre as medições de velocidade). Os grupos 3 e 5 obtiveram resultados melhores utilizando intervalo de $60 \mathrm{~s}$ entre as medições, ao invés de $30 \mathrm{~s}$.

Como explicado anteriormente, experimentalmente não se pode afirmar que qualquer redução do subintervalo usado para estimar o valor de uma integral por uma somatória, necessariamente faça com que o valor obtido pela somatória se aproxime do valor real desta integral, pois isso acontece de forma estatística e não individual; uma analogia pode ser feita pensando com o que acontece com a média de um experimento sujeito
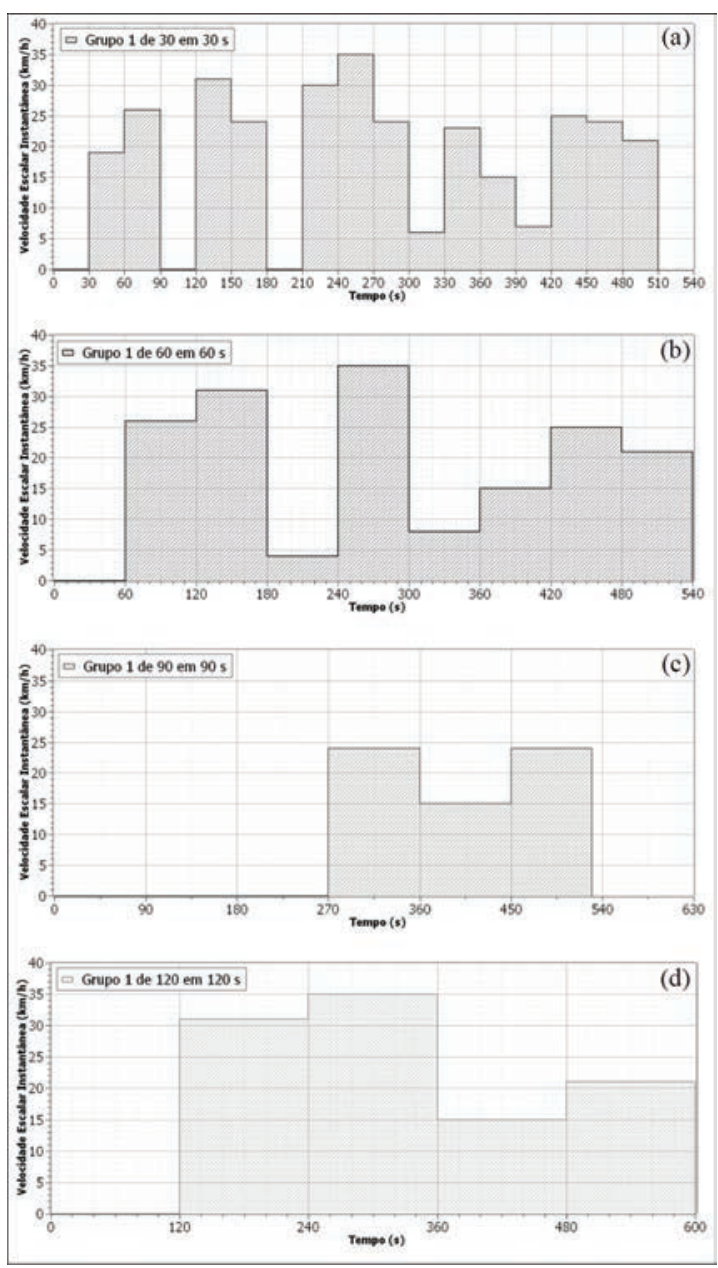

Figura 5: Retângulos aproximadores para $\circ$ Grupo 1, para os

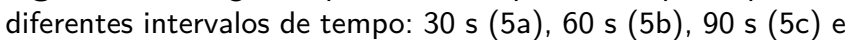
$120 \mathrm{~s}(5 \mathrm{~d})$. Este grupo utilizou as extremidades esquerdas dos subintervalos.

apenas a erros aleatórios: ao aumentar o número de dados, diminui-se a incerteza do valor médio, porém não é possível assegurar que o valor médio obtido de um experimento com mais dados esteja mais próximo do valor verdadeiro do que aquele obtido por um experimento com menos dados. Levando em conta essa condição, a análise dos resultados deve ser feita entre os diferentes grupos, utilizando os valores de aproximação da distância percorrida para intervalos de tempo iguais para calcular o valor médio e o desvio padrão amostral. Vale ressaltar que para essa análise estatística é preciso um número mínimo por volta de uma dezena de dados em cada intervalo de tempo entre medições $(30,60,90$ e $120 \mathrm{~s}$ ) e isso deve ser levado em conta no planejamento do experimento, talvez juntando dados de mais de uma sala de alunos, dependendo do número de alunos por sala.

A Tabela 3 apresenta uma análise estatística com os resultados de distância percorrida obtidos pelos grupos 1 a 4; uma vez que a análise comparativa das dispersões deve se basear em dados medidos em condições equivalentes, o grupo 5 ficou de fora dessa análise 
Tabela 2: Resultados das distâncias percorridas calculadas pelos grupos, em intervalos padronizados de tempo.

\begin{tabular}{lccc}
\hline Grupo & $\begin{array}{c}\text { Intervalo de } \\
\text { tempo entre } \\
\text { medidas (s) }\end{array}$ & $\begin{array}{c}\text { Número de } \\
\text { pontos } \\
\text { coletados }\end{array}$ & $\begin{array}{c}\text { Distância } \\
\text { total } \\
\text { calculada }(\mathrm{km})\end{array}$ \\
\hline $\mathbf{1}$ & 30 & 18 & 2,6 \\
& 60 & 10 & 2,8 \\
$\mathbf{2}$ & 90 & 7 & 1,6 \\
& 120 & 6 & 3,4 \\
& 30 & 25 & 2,6 \\
$\mathbf{3}$ & 60 & 12 & 2,7 \\
& 90 & 9 & 1,0 \\
& 120 & 8 & 1,9 \\
& 30 & 24 & 2,0 \\
$\mathbf{4}$ & 60 & 14 & 2,5 \\
& 90 & 9 & 2,7 \\
& 120 & 8 & 2,9 \\
& 30 & 27 & 2,8 \\
$\mathbf{5}$ & 60 & 14 & 3,5 \\
& 90 & 9 & 1,5 \\
& 120 & 8 & 3,9 \\
& 30 & 12 & 1,6 \\
& 60 & 7 & 2,4 \\
& 120 & 5 & 0,68 \\
& & 4 & 1,00 \\
\hline & & &
\end{tabular}

Tabela 3: Valores médios de distância percorrida, desvio padrão amostral e desvio padrão da média com resultados obtidos apenas pelos grupos de 1 a 4 .

\begin{tabular}{cccc}
\hline $\begin{array}{c}\text { Intervalo } \\
\text { de tempo } \\
\text { entre } \\
\text { medições }(\mathrm{s})\end{array}$ & $\begin{array}{c}\text { Valor } \\
\text { médio de } \\
\text { distância } \\
\text { percorrida }(\mathrm{km})\end{array}$ & $\begin{array}{c}\text { Desvio } \\
\text { padrão } \\
\text { amostral } \\
(\mathrm{km})\end{array}$ & $\begin{array}{c}\text { Desvio } \\
\text { padrão } \\
\text { da média } \\
(\mathrm{km})\end{array}$ \\
\hline 30 & 2,50 & 0,31 & 0,16 \\
60 & 2,88 & 0,42 & 0,21 \\
90 & 1,70 & 0,70 & 0,35 \\
120 & 3,01 & 0,84 & 0,42 \\
\hline
\end{tabular}

por apresentar anomalia nos dados, conforme discutido anteriormente.

\section{Análise dos Resultados Aprimorados e Conclusões}

Comparando os valores médios de distância percorrida e as dispersões de valores obtidos nessas estimativas em função do passo utilizado (Tabela 3) pode-se afirmar, apesar do número pequeno de dados usado na análise, que à medida que reduzimos o tempo entre medições (ou, equivalentemente, aumentamos o número de subintervalos) há uma melhora do valor de distância percorrida calculada pela somatória das áreas dos retângulos aproximadores. Adicionalmente, o valor médio obtido de distância percorrida para o menor passo utilizado é mais próximo do valor real (na verdade é coincidente), porém não se pode garantir que isso ocorra sempre, ainda que com um bom número de dados para uma boa análise estatística.

Outro fator importante para o planejamento do experimento é a escolha do trecho percorrido pelo automóvel; em um trecho urbano há grande interferência de semáforos, engarrafamentos, pontos de parada no caso de ônibus, e também um limite baixo de velocidade máxima permitida. Em um trecho de estrada percorrido por automóvel de passeio, por outro lado, não há semáforos, o limite de velocidade máxima permitida é mais alto, possibilitando uma amplitude maior de valores obtidos de velocidade, e há menos paradas por engarrafamentos. Vale ressaltar que não há problema em realizar a medida de velocidade em instantes que o veículo esteja parado, pois a integral dá conta disso; o problema surge quando se toma dados aleatoriamente em uma distribuição de velocidades com um pico para velocidade nula, o que pode ocasionar uma grande diferença entre o valor calculado e o valor real de distância percorrida. $\mathrm{Na}$ condição de realização deste quase-experimento aqui reportado, a escolha do trecho e veículo se deu por questões práticas, pensando na padronização do trecho percorrido entre duas unidades sem parada para subida e descida de passageiros, na condição socioeconômica de muitos estudantes que não podem contar com um automóvel para a realização da atividade, assim como na segurança desses estudantes, evitando que algum aluno se pusesse a coletar os dados de velocidade enquanto dirigia.

\section{Considerações Finais}

A atividade mostrou-se muito eficiente para os objetivos da proposta. Alguns estudantes tiveram dificuldade na realização dos cálculos das áreas de cada retângulo aproximador, mostrando que realmente não compreendiam o significado do que estavam calculando; mesmo obtendo resultados muito discrepantes, não foram capazes de voltar aos dados coletados e tentar resolver o problema sozinhos; essas dúvidas foram sanadas na discussão posterior em sala de aula, e em vista disso sugerese que seria mais eficiente se todos os alunos pudessem fazer a coleta de dados de velocidade e tempo e depois realizarem os cálculos e os retângulos aproximadores em sala de aula em conjunto com o docente. Apesar de algumas dificuldades encontradas para os cálculos, a maioria dos estudantes esteve motivada pela atividade e curiosos (e alguns até descrentes) pelo fato que uma proposta tão simples de ser executada, coletando dados de velocidade e tempo, poderia encontrar a distância percorrida pelo ônibus através de aproximações de uma integral definida. Também alcançou-se com grande êxito que os estudantes pudessem compreender que a área $A$ (equações (1) e (5)), que foi calculada aproximadamente pela soma dos retângulos aproximadores, tem um significado físico, e nesta atividade era a distância percorrida pelo ônibus. Outro conceito importante trabalhado foi 
sobre a escolha dos pontos amostrais para as aproximações por retângulos; nenhum grupo escolheu o ponto médio dos subintervalos por causa da dificuldade adicional em calcular o valor médio da velocidade em cada subintervalo, porém como durante a trajetória do ônibus a função velocidade teve trechos crescentes seguidos por trechos decrescentes, alternadamente, trabalhar com os extremos direito, esquerdo ou o ponto médio não fez diferença significativa. Já se a função a ser integrada fosse estritamente crescente, ou estritamente decrescente, trabalhar com os pontos médios forneceria uma estimativa melhor do que com os pontos extremos.

Os resultados de distância percorrida obtidos independentemente pelos grupos se acercaram do valor real de $2,5 \mathrm{~km}$, porém nem sempre o valor estimado pelo intervalo de medição mais curto foi o melhor valor. Quando os dados dos cinco grupos foram tratados em conjunto, agrupados em função do intervalo de tempo entre medições, vê-se claramente que a aproximação é tanto melhor quanto menor seja o intervalo de tempo entre as medições, ou de forma equivalente, quanto mais retângulos aproximadores (maior $n$ ) sejam utilizados na estimativa da integral.

Dessa forma, o objetivo da atividade foi atingido, pois os estudantes perceberam através de uma vivência simples, a relação estreita entre o Cálculo e a Física, compreendendo o conceito matemático da soma infinita de áreas na integral definida para a estimativa de uma distância percorrida.

\section{Material suplementar}

O seguinte material suplementar está disponível online:

Material Suplementar - Histogramas de velocidades e retângulos aproximadores.

\section{Referências}

[1] G.L. Lima, Educação Matemática Pesquisa 16, 125 (2014).

[2] A. Chervel, História das disciplinas escolares: reflexões sobre um campo de pesquisa. Teoria ES Educação (Palmarinca, Porto Alegre, 1990), v. 2, p. 177.

[3] R.G.S. Miskulin e M.R.C. Silva, em: Tecnologia e Educação Matemática: ensino, aprendizagem e Formação de professores, editado por A.P. Jahn e N.S.G. Allevato (SBEM, Recife, 2010).

[4] F.S. Arista e H. Kuswanto, International Journal of Instruction 11, 1 (2018).

[5] T. Pierratos e H.M Polatoglou, Phys. Educ. 53, 015021 (2018).

[6] D. Bogusevschi, C.H. Muntean e G. Muntean, em: Society for Information Technology $\&$ Teacher Education International Conference (Las Vegas, 2019).

[7] J. Bonato, L.M. Gratton, P. Onorato e S. Oss, Phys. Educ. 52, 045017 (2017).
[8] J.C. Castro-Palacio, L. Velazquez, J.A. Gómez-Tejedor, F.J. Manjón e J.A. Monsoriu, Rev. Bras. Ensino Fís. 36, 1 (2014).

[9] R.S. Franco, G.F. Marranghello e F.S. Rocha, Rev. Bras. Ensino Fís. 38, 1308 (2016).

[10] A.G. Guedes, Rev. Bras. Ensino Fís. 37, 2502 (2015).

[11] C. Stampfer, H. Heinke e S. Staacks, Nature Reviews Materials 5, 169 (2020).

[12] D.J. O'Brien, American Journal of Physics 89, 403 (2021).

[13] D. Trowbidge e L.C. McDermott, Am. J. Phys. 48, 1020 (1980).

[14] D. Trowbidge e L.C. McDermott, Am. J. Phys. 49, 242, (1981).

[15] R.F.F. Cunha e D.G.G. Sasaki, Rev. Bras. Ensino Fís. 42, e20190149 (2020).

[16] C.B.C. Nascimento e A.L. Oliveira, Rev. Bras. Ensino Fís. 42, e20190162 (2020).

[17] J. Stewart, Cálculo: tradução da $7^{a}$ edição norteamericana (Cengage Learning, São Paulo, 2016), v. 1. 